\title{
Treatment of Drug-Induced Erythema Multiforme: Case Report
}

\author{
Hae-Ohk Lee ${ }^{1}$, Hye-Min Ju' ${ }^{1}$, Ji-Yeon Lee ${ }^{1}$, Hye-Mi Jeon ${ }^{2}$, Kyung-Hee Kim ${ }^{3}$, \\ Soo-Min Ok ${ }^{1}$, Yong-Woo Ahn ${ }^{1}$, Sung-Hee Jeong ${ }^{1}$ \\ 'Department of Oral Medicine, Dental Research Institute, Dental and Life Science Institute, \\ School of Dentistry, Pusan National University, Yangsan, Korea \\ ${ }^{2}$ Dental Clinic Center, Pusan National University Hospital, Busan, Korea \\ ${ }^{3}$ Department of Oral Medicine, Inje University Busan Paik Hospital, Busan, Korea
}

Received November 18, 2019 Revised December 11, 2019 Accepted December 11, 2019

\section{Correspondence to:}

Sung-Hee Jeong

Department of Oral Medicine, School of Dentistry, Pusan National University, 49

Busandaehak-ro, Mulgeum-eup, Yangsan

50612, Korea

Tel: +82-55-360-5242

Fax: +82-55-360-5234

E-mail:drcookie@pusan.ac.kr

https://orcid.org/0000-0002-6296-4775

\begin{abstract}
Erythema multiforme (EM) is an inflammatory immune-mediated mucosal disease. EM is classified as EM minor or EM major, depending on the severity of symptoms. Most patients with minor or major forms of EM have oral lesions. EM can occur as a result of adverse drug reactions but this is difficult to establish. Several indicators have been developed that can be used as a criterion of evaluation to ensure objectivity in identifying the causality of adverse drug reactions. The Naranjo algorithm was used in the evaluation to ensure objectivity in identifying the causality of adverse drug reactions. There were two cases of patients suspected of having EM induced by drugs. They were both assessed using the Naranjo algorithm to confirm the causality of the disease.
\end{abstract}

Key Words: Drug-related side effects and adverse reactions; Erythema multiforme; Rheumatoid arthritis

\section{INTRODUCTION}

Erythema multiforme (EM) is an inflammatory immunemediated disease that affects the skin or mucous membrane. It appears to be a hypersensitivity to infectious substances or drugs. Predominantly in healthy young adults, especially at the age of 20 to 40 . EM is a mucocutaneous condition characterized by blistering, ulcerative, and symmetrically distributed targets or iris lesions at the extremities and trunk [1].

EM is classified as EM minor or EM major, depending on the severity of symptoms such as mouth alone or skin eruption, or with oral or other mucosal lesions [2]. Oral EM is a distinct but not well recognized variant. The concept of oral EM is controversial and not universally accepted [1], but it is widely regarded by many authors as a distinct entity [3,4]. Diagnosis is often difficult because clinical symptoms mimic other oral inflammatory and vesiculobullous diseases.
Lesions of lip vermilion occur in some patients. Many authors still regard EM minor, EM major, Stevens-Johnson syndrome (SJS) and toxic epidermal necrolysis (TEN) as one disease continuum with a variety of clinical severity. Others regard EM as a separate entity in SJS and TEN, especially because of its strong association with infections such as herpes simplex virus (HSV) [5]. EM is mainly caused by exposure to microorganisms and is known to be caused in association with HSV. However, EM is also known to be caused by a number of drugs, such as antibiotics and nonsteroidal anti-inflammatory drugs [2].

The purpose of this study is to evaluate the diagnosis and treatment of EM in the oral cavity through a case report on drug-induced EM patients visiting the Department of Oral Medicine at Pusan National University Dental Hospital. 


\section{CASE REPORT}

\section{Case 1}

A 65-year-old male patient visited the Department of Oral Medicine at the Pusan National University Dental Hospital with the following complaints: his oral mucosa was sore and his lips were swollen for 2 days. On oral examination, extensive, irregular ulcerations with yellow base and erythematous borders on bilateral buccal mucosa, palate and hemorrhagic crusts were formed on the lips (Fig. 1). There were no premonitory symptoms of infection before the onset, but the patient mentioned that the drugs prescribed by the orthopedic surgeons for the treatment of rheumatoid arthritis (RA) were changed ten days earlier. The history of lip hemorrhagic crusting and rapid onset, as well as the history of medication changes were identified. C-reactive protein (CRP) was normal but erythrocyte sedimentation rate (ESR) and rheumatoid factor were elevated. The HSV antibody IgM levels were negative. Liver function tests (LFT), renal function tests (RFT) were normal. Based on the following clinical findings and history, the primary diagnosis was Leflunomide-induced EM. A corticosteroid gargle (0.06\% Prednisolone solution) was applied 5 to 10 minutes for 1 time, three times daily for the treatment of the lesion. The patient asked the orthopedic surgeon whether if the suspected drug could be discontinued or replaced. Because RA is favorable, leflunomide was stopped, which is known to cause EM. A week later, the patient did not show any significant improvement of the lesion during the second visit to the Dental Hospital. Therefore, corticosteroid (Solondo Tab., Yuhanmedica, Seoul, Korea) was administered orally in tapering doses $(30 \mathrm{mg}$ prednisolone tapered down by $5 \mathrm{mg}$ everyday) and systemic corticosteroids (Dexamethasone Disodium Phosphate $5 \mathrm{mg} / \mathrm{mL}$ ) were injected directly into circulation (IV). Corticosteroid gargle was maintained, and topical antimycotics (PMS-Nystatin suspension; Pharmascience Inc., Montreal, QU, Canada) was used as gargle solution ( $5 \mathrm{~mL}$ ) 5 to 10 minutes for 1 time, three times daily to prevent side effects such as candidiasis. A week after the second visit, the patient was examined again and a decrease in hemorrhagic crusts on lips and extensive ulceration of the buccal mucosa were observed. Corticosteroid gargle and topical antimycotics use remained the same for 14 days and symptoms improved at the fourth
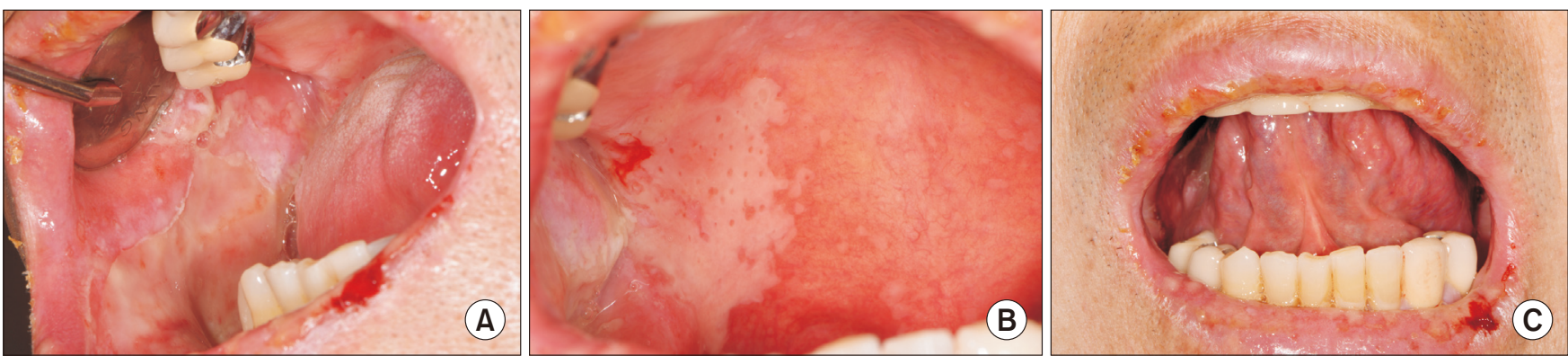

Fig. 1. Case 1: Oral mucosal, palate and lip lesions at first visit. (A) Extensive ulceration on right buccal mucosa. (B) Palatal ulcerations with irregular border. (C) Blood encrusted lip lesions.
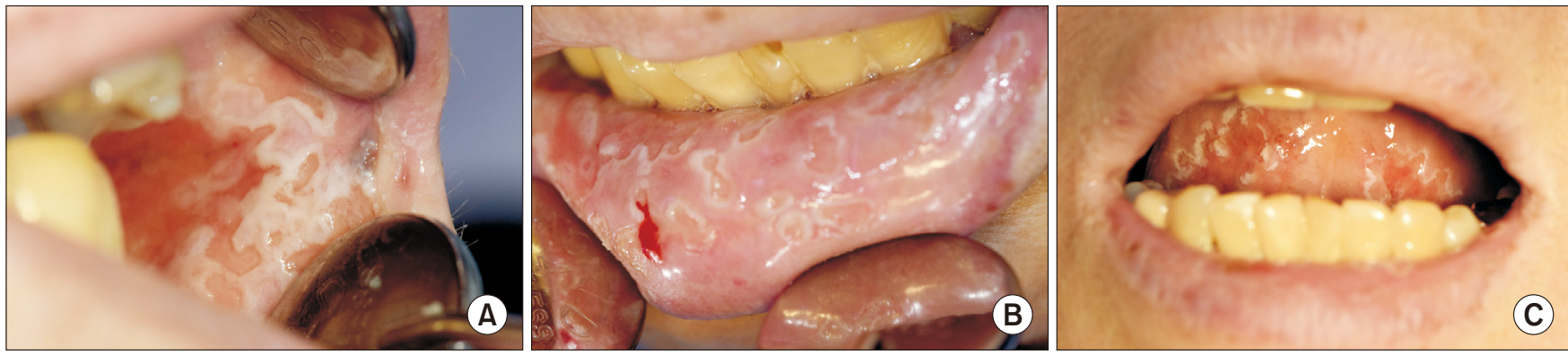

Fig. 2. Case 2: Oral mucosal, lip and tongue lesions at first visit. (A) Extensive erosive ulceration with irregular white border on left buccal mucosa. (B) Multiple small ulcers with irregular border on lower lip. (C) Erythematous ulcers on ventral side of the tongue. 
visit two weeks later.

\section{Case-2}

A 70-year-old female patient visited the Department of Oral Medicine at the Pusan National University Dental Hospital with complaints that it was difficult to eat, drink, and swallow with a number of ulcers in the mouth. Clinical findings showed a number of ulcers on the oral mucosa including buccal mucosa, labial mucosa and the ventral side of the tongue (Fig. 2). The patient complained of severe oral mucosal pain when opening the mouth, making it difficult to eat, drink and swallow. The patient needed to be hospitalized and required supportive care, so was referred to the Department of Oral and Maxillofacial Surgery. Her hematological investigation showed elevated CRP, abnormal LFT and RFT and serum electrolytes. The primary diagnosis was EM with extensive ulcers and rapid onset. At the time of onset, a review was performed to determine whether there were any remarkable diseases or special medications that could cause the lesion and it was confirmed that methotrexate and tacrolimus were used to treat RA in the patient. Consultation with a dermatologist was performed to ensure accurate assessment of the symptoms. Methotrexate was ceased as it was a possible cause of EM in the patient. The symptoms appeared to subside after methotrexate was discounted, suggesting the drug was indeed the cause of EM. Corticosteroid (Dexamethasone Disodium Phosphate $5 \mathrm{mg} /$ $\mathrm{mL}$ ) injection was used three times daily into the circulation (IV) for 3 days as both a treatment and to manage the condition. At 10 days of hospitalization, symptoms disappeared and the patient was discharged and on follow up of 1 month post discharge there was no recurrence observed.

\section{DISCUSSION}

EM is associated with several etiologies, including drugs, malignant tumors and sarcoidosis, but about 90\% of them are associated with infectious agents, such as HSV for adults and Mycoplasma Pneumonia for children. Most patients with minor or major forms of EM (70\%) have oral lesions [6]. In the case of EM minor, mucosal lesions are infrequent but mainly appear in the oral mucosa. EM major lesions are known to appear in more than two areas of the genital, ocular, and laryngeal mucosa, including the oral mucosa. It has also been reported to be expressed only in the oral cavity without skin lesions [4]. Oral involvement may occur before other lesions in the other stratified squamous epithelia or may occur alone [6]. Lesions affecting the oral mucosa mainly appear on the lips, buccal mucosa, and tongue, and typically appear as hemorrhagic crusts of the lips [3]. There can be severe pain in the oral cavity and surrounding areas that can impair language, diet, and fluid intake. Lips and mouth lesions heal without scarring. Recurrence can be seen in about 25\%. Periodicity may vary, attacks last between 10 to 20 days, once or twice a year, and are usually resolved after about 6 times within an average of 10 years [7].

Available diagnostic laboratory tests do not help to diagnose EM. Signs of laboratory abnormalities, such as increased ESR, white blood cell counts, and liver enzyme levels, can be seen in severe cases [1]. The diagnosis of EM is mainly based on the patient history and the clinical presentation because the histopathologic features and laboratory investigations are non-specific. Typically, history includes acute onset of oral and/or skin diseases, and a history of HSV infection or recent drug intake preceeding the onset. This history, combined with the characteristic target lesions of the skin and mucous membrane, supports the diagnosis of EM. Direct and indirect immunofluorescence can help distinguish between different types of vesiculo-bullous lesion and EM. Tzanck smear, swab for HSV-PCR or other serological investigations may help to rule out other inflammatory, autoimmune or malignant diseases [8].

The pathogenesis pathway of EM is based on the research studies of HSV associated EM (HAEM). Autoreactive T-cell triggers by viral antigen-positive cells containing the HSVDNA polymerase gene have been suggested to play an important role [9]. EM is initiated by the expression of HSVDNA fragments, probably transported and produced by peripheral blood mononuclear cell, mainly macrophages and CD34+ Langerhans cell precursors, possibly by the vascular pathway. The inflammatory responses are initiated by viral gene expression in the skin and recruitment of HSV-specific CD4+ Th1 cells. Interferon- $\gamma($ IFN- $\gamma)$ generated by this reaction upregulates cytokines and chemokines to amplify skin inflammatory events through increased sequestration 
of circulating leukocytes, monocytes and NK cells and recruitment of autoreactive T-cells to the epidermis [9]. The mechanism of generation of autoreactive T-cells is still unclear. The result is epidermal damage caused by lysis of peripheral keratinocytes, release of various cytotoxic factors, keratinocyte growth arrest and apoptosis. In drug-induced EM (DIEM), the reactive metabolites of the initiating drug are thought to induce disease [10], but the mechanism of injury is variable and unlike HAEM, does not appear to be the result of the delayed-type hypersensitivity reaction. Immunocytochemical staining and in situ hybridization showed that $\mathrm{T}$ cells do not produce IFN- $\gamma$ in drug-induced lesions, but rather the lesions are characterized by TNF- $\alpha$ present in keratinocytes and produced by macrophages and monocytes. In contrast, TNF- $\alpha$ was not detected in HAEM and it was suggested that it can be used as a laboratory test to distinguish drug-induced lesions from HAEM [9]. Locally produced TNF- $\alpha$ can be important because it has been shown to mediate keratinocyte apoptosis [11], and this mechanism may play an important role in mild forms of DIEM.

EM can occur as a result of adverse drug reactions caused by medicines used for the diagnosis, treatment or prevention of diseases. Therefore, efforts to collect and evaluate information on adverse drug cases and to reveal causal relationships between adverse drug cases and drugs can contribute to reducing the damage of adverse drug reactions [12]. When an adverse drug reaction is suspected, it is difficult to determine whether the response is caused by a drug or, which drug is responsible for this reaction, but it is essential to systematically manage the adverse drug reaction [13]. Several indicators have been developed that can be used as a criterion of evaluation to ensure objectivity in identifying the causality of adverse drug reactions [12]. Such algorithms include the World Health Organization-Uppsala Monitoring Center causality categories [13], Naranjo algorithm [14], Kramer algorithms [15], etc. The Naranjo algorithm used in this study based on 10 question scoring system (Table 1) [16]. The 10 questions address factors such as: the temporal causal relationship between drug administration and adverse drug reaction, the patient's response after discontinuation or re-administration of the drug, the assessment of the likely cause of the patient's underlying disease, and the presence of similar reactions to past drugs, response to placebo, drug concentration measurement, and objective testing to support adverse drug cases [14].

The likelihood of adverse reactions associated with drug therapy depends on the sum of the points given for each answer to a question that can be answered as positive (yes), negative (no) or unknown or not applicable (unknown). It is classified as definite, probable, possible, doubtful. The adverse drug reaction was assigned to the probability category in the total score as follows: Definite $\geq 9$, probable 5-8, possible 1-4, doubtful $\leq 0$ [14].

In the first case, symptoms occurred after the patient started taking the leflunomide and, symptoms subside after the drug was discontinued. There was no evidence of any other causes. Naranjo algorithm showed a probable drug cause score of $6+$, indicating that the symptoms were

Table 1. Naranjo adverse drug reaction probability scale [16]

\begin{tabular}{|c|c|c|c|}
\hline Question & Yes & No & Don't know \\
\hline 1. Are there previous conclusive reports on this reaction? & +1 & 0 & 0 \\
\hline 2. Did the adverse reaction appear after the suspected drug was administered? & +2 & -1 & 0 \\
\hline $\begin{array}{l}\text { 3. Did the adverse reaction improve when the drug was discontinued or } \\
\text { a specific antagonists was administered? }\end{array}$ & +1 & 0 & 0 \\
\hline 4. Did the adverse reaction reappear when the drug was readministered? & +2 & -1 & 0 \\
\hline 5. Are there alternative causes that could on their own have caused the reaction? & -1 & +2 & 0 \\
\hline 6. Did the reaction reappear when a placebo was given? & -1 & +1 & 0 \\
\hline 7. Was the drug detected in the blood (or other fluids) in concentrations known to be toxic? & +1 & 0 & 0 \\
\hline 8. Was the reaction more severe when the dose was increased or less severe when the dose was decreased? & +1 & 0 & 0 \\
\hline 9. Did the patient have a similar reaction to the same or similar drug in any previous exposure? & +1 & 0 & 0 \\
\hline 10. Was the adverse event confirmed by any objective evidence? & +1 & 0 & 0 \\
\hline
\end{tabular}

Scoring: $\geq 9$ : definite, 5-8: probable, 1-4: possible, $\leq 0$ : doubtful. 
significantly related to the medication. This is also consistent with several previous studies that reported cases of EM being caused by leflunomide. In the second case, many adverse reactions due to methotrexate were reported, and symptoms were resolved after withdrawal of the drug. There were no other causes such as an infection observed. Naranjo's algorithm showed a possible drug cause score of 4+, which classified it as possible and indicates that the symptoms may be related to the drug.

In the treatment of EM, it is important to identify and eliminate the triggers of the disease. If infection is considered a cause, it is necessary to use an antiviral agent or an antibiotic as treatment for the causative virus or bacteria. If a particular drug is identified as the causal agent, it needs to be ceased immediately. Self-defining features can be used as an indicator of improvement of symptoms after the causative agent is removed [2]. Corticosteroids are the most commonly used drugs for EM management despite the lack of evidence. Treatment of mild diseases should focus on alleviating the symptoms with topical anti-inflammatory agents, anesthetics or painkillers [8]. In severe forms of EM, there may be extensive lesions or lesions that make it difficult for food intake. Depending on the etiology and severity of the disease, a systemic steroid may be recommended in consultation with the doctor. The most commonly used steroid is 40 to $60 \mathrm{mg}$ of prednisone per day tapered over 2 to 4 weeks. Supportive treatment should be provided in the form of liquid diets, intravenous fluids, electrolytes and nutritional support [8].

In this case, the intraoral bullous, ulcerative lesions and hemorrhagic crusts of the lips were diagnosed and treated as EM, caused by leflunomide and methotrexate administered for treatment of RA. The diagnosis was based on clinical findings such as hemorrhagic crusts, rapid onset, and widespread ulcerative lesions in the oral cavity, and recent medical history with new drugs. An analysis of the literature shows that cases of leflunomide-induced EM expression were introduced in 2003 by Fischer et al. [17], and cases of methotrexate-induced EM expression were introduced in 2011 by Omoregie et al. [18]. These studies report treatment regimens consisting of discontinuation of the suspected causal drug and the use of steroids. Even though the primary attack of oral EM is confined to the oral mucous membrane, subsequent attacks can produce a more serious form of EM involving the skin. Therefore, it is important to distinguish the oral EM for early diagnosis, prompt management and proper follow-up.

\section{CONFLICT OF INTEREST}

No potential conflict of interest relevant to this article was reported.

\section{ORCID}

\author{
Hae-Ohk Lee \\ https://orcid.org/0000-0002-9859-5342 \\ Hye-Min Ju \\ https://orcid.org/0000-0002-9252-6717 \\ Ji-Yeon Lee \\ https://orcid.org/0000-0001-9654-5723 \\ Hye-Mi Jeon \\ https://orcid.org/0000-0003-0007-5662 \\ Kyung-Hee Kim \\ https://orcid.org/0000-0003-2922-6452 \\ Soo-Min Ok \\ https://orcid.org/0000-0003-1776-371X \\ Yong-Woo Ahn \\ https://orcid.org/0000-0002-2197-0394 \\ Sung-Hee Jeong \\ https://orcid.org/0000-0002-6296-4775
}

\section{REFERENCES}

1. Huff JC, Weston WL, Tonnesen MG. Erythema multiforme: a critical review of characteristics, diagnostic criteria, and causes. J Am Acad Dermatol 1983;8:763-775.

2. Scully C, Bagan J. Oral mucosal diseases: erythema multiforme. Br J Oral Maxillofac Surg 2008;46:90-95.

3. Bean SF, Quezada RK. Recurrent oral erythema multiforme. Clinical experience with 11 patients. JAMA 1983;249:2810-2812.

4. Lozada-Nur F, Gorsky M, Silverman S Jr. Oral erythema multiforme: clinical observations and treatment of 95 patients. Oral Surg Oral Med Oral Pathol 1989;67:36-40.

5. Côté B, Wechsler J, Bastuji-Garin S, Assier H, Revuz J, Roujeau JC. Clinicopathologic correlation in erythema multiforme and Stevens-Johnson syndrome. Arch Dermatol 1995;131:1268-1272.

6. Farthing P, Bagan JV, Scully C. Mucosal disease series. Number IV. Erythema multiforme. Oral Dis 2005;11:261-267. 
7. French LE, Prins C. Erythema multiforme, Stevens-Johnson Syndrome and toxic epidermal necrolysis. In: Bolognia JL, Jorizzo JL, Rapini RP, et al, eds. Dermatology. 2nd ed. London: Mosby Elsevier; 2008. pp. 287-300.

8. Samim F, Auluck A, Zed C, Williams PM. Erythema multiforme: a review of epidemiology, pathogenesis, clinical features, and treatment. Dent Clin North Am 2013;57:583-596.

9. Aurelian L, Ono F, Burnett J. Herpes simplex virus (HSV)-associated erythema multiforme (HAEM): a viral disease with an autoimmune component. Dermatol Online J 2003;9:1.

10. Knowles SR, Uetrecht J, Shear NH. Idiosyncratic drug reactions: the reactive metabolite syndromes. Lancet 2000;356:1587-1591.

11. Paul C, Wolkenstein P, Adle H, et al. Apoptosis as a mechanism of keratinocyte death in toxic epidermal necrolysis. Br J Dermatol 1996;134:710-714.

12. Meyboom RH, Hekster YA, Egberts AC, Gribnau FW, Edwards IR. Causal or casual? The role of causality assessment in pharmacovigilance. Drug Saf 1997;17:374-389.
13. Edwards IR, Aronson JK. Adverse drug reactions: definitions, diagnosis, and management. Lancet 2000;356:1255-1259.

14. Naranjo CA, Busto U, Sellers EM, et al. A method for estimating the probability of adverse drug reactions. Clin Pharmacol Ther 1981;30:239-245.

15. Kramer MS, Leventhal JM, Hutchinson TA, Feinstein AR. An algorithm for the operational assessment of adverse drug reactions. I. Background, description, and instructions for use. JAMA 1979;242:623-632.

16. Son MK, Lee YW, Jung HY, et al. Comparison of the naranjo and WHO-uppsala monitoring centre criteria for causality assessment of adverse drug reactions. Korean J Med. 2008;74:181-7.

17. Fischer TW, Bauer HI, Graefe T, Barta U, Elsner P. Erythema multiforme-like drug eruption with oral involvement after intake of leflunomide. Dermatology 2003;207:386-389.

18. Omoregie FO, Ukpebor M, Saheeb BD. Methotrexate-induced erythema multiforme: a case report and review of the literature. West Afr J Med 2011;30:377-379. 